\title{
El arbitraje médico y las quejas concluidas relacionadas con la población de menores de 15 años durante el periodo 2011-2015
}

\author{
Sonia B. Fernández-Cantón* y José N. Rizo-Amézquita \\ Dirección General de Difusión e Investigación de la Comisión Nacional de Arbitraje Médico, Ciudad de México, México
}

\begin{abstract}
Resumen
Introducción: Las quejas constituyen un indicador negativo en la calidad de la atención médica. El conocimiento de los problemas que las originan y sus consecuencias constituyen elementos fundamentales para las estrategias de mejora de la calidad. En este estudio se dan a conocer los resultados del análisis de laudos concluidos, emitidos por la Comisión Nacional de Arbitraje Médico durante el periodo 2011-2015, que corresponden a la población usuaria menor de 15 años. Métodos: Estudio transversal descriptivo. Se analizó información extraída de los laudos concluidos en los últimos 5 años en los que la población afectada tuviera menos de 15 años, independientemente del servicio involucrado. Resultados: Se detectaron 40 laudos, la mayoría involucrados con servicios de pediatría, encontrándose una distribución homogénea por edad y sexo. Los porcentajes más altos se encontraron en las edades extremas de la población estudiada: menores de 1 año (32\%) y de 10 a 14 años (30\%). La principal causa de la queja fueron los problemas con los auxiliares de diagnóstico (45\%). Se detectó un promedio de 8.7 eventos adversos por caso, la mayoría relacionados con procedimientos. Entre los daños destacan 12 defunciones y 9 discapacidades permanentes. Se encontró evidencia de mala práctica en el 65\% de los casos y hubo una sentencia condenatoria en el 60\% de los laudos. Conclusiones: Se obtuvieron resultados en cuatro grandes apartados: características sociodemográficas, descripción de la atención médica, eventos adversos y sus repercusiones en los daños a la salud, y la evaluación del acto médico y conclusiones del laudo.
\end{abstract}

Palabras clave: Controversias médicas. Quejas. Arbitraje médico. Mala práctica. Eventos adversos.

\section{Medical arbitration and claims for malpractice related to health service users under 15 years of age during 2011-2015}

\section{Abstract}

Background: Claims by health services users are a negative indicator of the care quality. To know the situations that originate them, and their consequences are key issues to consider in order to design strategies to improve the quality of health services. This study analyses the cases submitted to the National Medical Arbitration Commission with a final arbitration decision during the period 2011-2015, related to health service users younger than 15 years old. Methods: Cross-sectional descriptive study. We analyzed information extracted from the reports with a final arbitration decision in the last 5 years,

\section{Correspondencia:}

*Sonia B. Fernández Cantón

E-mail: sonia_fernandez@prodigy.net.mx
Fecha de recepción: 18-08-2017

Fecha de aceptación: 03-12-2017

DOI: 10.24875/BMHIM.M18000017
Disponible en internet: 14-05-2018 Bol Med Hosp Infant Mex. 2018;75:183-193 www.bmhim.com

1665-1146/@ 2018. Hospital Infantil de México Federico Gómez, impreso por Permanyer México SA de CV, todos los derechos reservados. 
selecting those where the affected users were younger than 15 years old. Results: $A$ total of 40 cases arbitral award were found, most of them involving pediatric services, with a similar number of male and female users and a similar proportion in the extreme age groups, $<1$ year (32\%) and 10-14 years (30\%). The main cause of the complaint was related to diagnostic procedures (45\%). An average of 8.7 adverse events per case were identified, most of them associated with procedures. Among the damages, there were 12 deaths and 9 permanent disabilities. There was evidence of malpractice in $65 \%$ of the cases and in $60 \%$ of the cases, the sentence was condemnatory. Conclusions: This manuscript shows information in four main sections: sociodemographic characteristics, description of medical care, adverse events and their repercussions on health damages, evaluation of the medical act and conclusions of the arbitration process.

Key words: Medical controversies. Claims. Medical arbitration. Malpractice. Adverse events.

\section{Introducción}

En este trabajo se pretende abordar la importancia de la queja médica en relación con los procesos de mejora de la calidad de la atención y el papel fundamental que para tal efecto ha desempeñado, desde su creación, la Comisión Nacional de Arbitraje Médico (CONAMED). En efecto, desde 1996, esta institución tiene como función principal ofrecer mecanismos alternativos para la solución de controversias derivadas de la atención a la salud, lo que representa ventajas importantes para quien se ve involucrado en este tipo de conflictos.

Si bien la mayoría de las quejas médicas vinculadas a los servicios de pediatría se resuelven a través del proceso conciliatorio, es importante abordar, de manera complementaria, lo que sucede con aquel otro segmento de quejas (aproximadamente el $3 \%$ ). En estos casos, al no ser resueltas durante la fase de la conciliación, los interesados deciden optar por el arbitraje médico y dejar en manos de un perito institucional la resolución del conflicto.

En ese contexto se aborda el análisis de las quejas médicas que se resolvieron a través de la emisión de un laudo a durante el periodo 2011-2015, independientemente del carácter de la sentencia dictada. Los resultados hacen referencia al proyecto ARIEL (Automatización de Registros de Información Estadística sobre Laudos) ${ }^{1}$, correspondiente a esos años para la población usuaria demandante de menores de 15 años.

Es importante destacar que las quejas que se resuelven a través del arbitraje médico representan los casos de mayor complejidad, los cuales no pudieron ser resueltos mediante los otros mecanismos alternativos ofrecidos por la CONAMED (asesorías, gestiones inmediatas, conciliaciones, etc.). En ese sentido, su

a Se entiende por laudo la resolución final especializada pronunciada por el perito arbitral (en este caso CONAMED), mediante la cual se pone fin a la controversia entre las dos partes involucradas. análisis representa, en forma significativa, el impacto negativo de la calidad de la atención médica sobre la seguridad del paciente.

Como antecedente genérico del análisis que se propone realizar, es conveniente señalar que, hasta hace algunos años, el abordaje de los problemas de salud de toda la población se había realizado, casi en forma tradicional, a partir de la información estadística de la morbilidad y la mortalidad registrada por las instituciones de salud. No obstante, cada vez con mayor claridad, la realidad muestra que las medidas obtenidas a partir de tales categorías son ahora insuficientes para describir los problemas sanitarios cada vez más complejos en los que se encuentra inmersa nuestra sociedad $^{2}$. En ese sentido, se enmarcan conceptos relativamente novedosos (al menos en su cuantificación) para el conocimiento de la calidad de la atención y la queja médica, esta última como indicador negativo de la primera, para cuyo análisis y evaluación se requieren nuevas estadísticas que documenten dichos aspectos. Tal es el caso, por ejemplo, de las cifras sobre los incidentes adversos que se ha logrado integrar a partir de la Clasificación Internacional de Seguridad del Paciente, propiciando una nueva categoría de información.

Cabe destacar que poco se sabe de las motivaciones que llevan a la población a presentar o no una queja. Sin embargo, existe cierto consenso en mencionar que su presentación se da, en gran medida, por factores tales como la opinión de otro médico al descalificar a colegas en cuanto a su actuación, la presencia de una sociedad más exigente y mejor informada, el papel desempeñado por ciertos medios de comunicación y la cada vez más creciente industria de litigios contra médicos ${ }^{3}$.

Es de destacar que las quejas atendidas en el contexto del arbitraje médico son particularmente valiosas porque permiten conocer tres dimensiones del problema bajo un enfoque múltiple: 1) el del usuario de los servicios, a partir del relato de hechos entregado al 
momento de presentar la queja; 2) el del prestador de servicios de salud, a partir de la documentación probatoria de la atención brindada; y 3) la del perito institucional de CONAMED, a partir de la revisión documental especializada e imparcial desde un punto de vista técnico médico.

El objetivo de este reporte es dar a conocer los resultados encontrados en el análisis de los 40 expedientes de laudos concluidos que han sido emitidos por la CONAMED durante el periodo 2011-2015, correspondientes a la población usuaria menor de 15 años, con particular énfasis en lo referente a los incidentes adversos.

La premisa implícita de este propósito es que el no conocer nuestra realidad conduce a no tomar conciencia de los riesgos existentes en cuanto a la ocurrencia de incidentes adversos, y por tanto a la no implementación de medidas de mejora de la calidad de los servicios de salud, lo que implica no trabajar en la prevención de los daños a los pacientes.

\section{Métodos}

Si bien la CONAMED cuenta con un sistema institucional de registro de datos, este es de carácter administrativo y su función es dar seguimiento a los casos atendidos en las diversas fases del proceso, por lo que las bases de datos así obtenidas no necesariamente permiten contar con el nivel de desagregación deseado de las diferentes variables y categorías de análisis. Por ello, a finales de 2014 se inició la implementación del proyecto ARIEL, el cual consiste en la revisión y el análisis de las quejas concluidas mediante la emisión de un laudo.

El proceso inicia con la revisión de los expedientes conformados en ocasión del levantamiento formal de la queja; a partir de un formato diseñado en forma expresa, la información susceptible de ser cuantificada es codificada a partir de catálogos definidos ${ }^{4}$ (entre los cuales tres son de carácter internacional y varios son nacionales, sectoriales e institucionales) ${ }^{\mathrm{b}}$, de tal forma que se genera una estadística sólida sobre los laudos concluidos. A la fecha, se cuenta con resultados de 2011 a 2015, sumando aproximadamente 350 laudos

b Incluye la Clasificación Internacional de Enfermedades (CIE-10), de Procedimientos (CIE_9MC) y de Seguridad del Paciente (CISP); los Catálogos Nacionales de Integración Territorial del INEGI (localidades, municipios y entidades federativas), Catálogo de Ocupaciones, de Discapacidad, de Especialidades Médicas (SEP/DGP), y de Establecimientos de Salud (SSA/CLUES), entre las más relevantes. analizados, y de ellos, 40 se refieren a usuarios menores de 15 años. Cabe mencionar que los laudos incluyen quejas provenientes tanto del sector privado como del público (a excepción del Instituto Mexicano del Seguro Social, por ser una institución que no se somete al proceso arbitral que realiza la CONAMED, bajo el argumento de que cuenta con los recursos legales para la resolución de conflictos al interior de la propia institución $)^{5}$.

Para este análisis se realizó un estudio de tipo descriptivo y transversal que incluye las principales variables captadas en el marco conceptual del proyecto mencionado. El manejo estadístico se llevó a cabo a partir de bases de datos en Excel $^{\circledR}$, de las cuales se obtuvieron frecuencias simples, distribuciones porcentuales, proporciones y razones. En todo momento se aseguró la confidencialidad de los datos personales, tal como señala la legislación correspondiente.

\section{Resultados}

La estructura del artículo responde al Modelo Mexicano de Arbitraje Médico ${ }^{6}$, el cual aborda las diferentes facetas a considerar en el análisis de la queja: las características sociodemográficas del usuario, las características de los establecimientos médicos involucrados y la atención a la salud, los daños de la población usuaria y los motivos de la queja, los eventos adversos y la seguridad del paciente, así como el desempeño institucional respecto a la evaluación del acto médico y la conclusión del laudo.

\section{Características sociodemográficas de la población usuaria}

En términos generales, la mayoría de los usuarios atendidos en los procesos arbitrales médicos de la institución son mujeres. En el presente estudio, de los 40 laudos que fueron emitidos durante el periodo analizado, se observó un comportamiento totalmente equitativo en cuanto al sexo: 20 de sexo masculino y 20 de sexo femenino. La distribución por edad también muestra una distribución homogénea, con los porcentajes más altos en las edades extremas de este segmento de población: menores de 1 año (32.5\%) y de 10 a 14 años $(30 \%)$, y muy similares en los otros dos grupos de edad (tabla 1).

En cuanto a la entidad de residencia, la población demandante declaró residir en alguna de las 12 entidades federativas del país, de tal forma que, como era de esperar, la mayoría declaró vivir en la Ciudad de 
Tabla 1. Distribución por edad y sexo de los usuarios de servicios de salud menores de 15 años correspondientes a los laudos del periodo 2011-2015

\begin{tabular}{|l|c|c|c|c|}
\hline \multirow{2}{*}{ Grupos de edad } & \multicolumn{2}{|c|}{ Sexo } & \multirow{2}{*}{ Total } & Porcentaje \\
\cline { 2 - 5 } & Masculino & Femenino & & 32.5 \\
\hline Menos de 1 año & 8 & 5 & 13 & 17.5 \\
\hline $1-4$ años & 3 & 4 & 7 & 20 \\
\hline $5-9$ años & 5 & 3 & 8 & 30 \\
\hline $10-14$ años & 4 & 8 & 12 & 100 \\
\hline Total & 20 & 20 & 40 & 20 \\
\hline
\end{tabular}

Tabla 2. Institución involucrada en la queja médica según tipo de establecimiento, periodo 2011-2015

\begin{tabular}{|c|c|c|c|c|c|}
\hline \multirow[t]{2}{*}{ Institución* } & \multicolumn{3}{|c|}{ Tipos de establecimiento } & \multirow[t]{2}{*}{ Total } & \multirow[t]{2}{*}{ Porcentaje } \\
\hline & Consulta externa & Hospitalización & De apoyo & & \\
\hline SSA & 1 & 6 & & 7 & 17.5 \\
\hline ISSSTE & 1 & 6 & & 7 & 17.5 \\
\hline Estatal & - & 2 & - & 2 & 5 \\
\hline Privado & 10 & 13 & 1 & 24 & 60 \\
\hline Total & 12 & 27 & 1 & 40 & 100 \\
\hline
\end{tabular}

ISSSTE: Instituto de Seguridad y Servicios Sociales de los Trabajadores del Estado; SSA: Secretaría de Salud.

*El Instituto Mexicano del Seguro Social, quien presenta el mayor volumen de quejas totales, no se somete al proceso arbitral de la CONAMED.

México (18 casos, $45 \%$ de los usuarios), siguiendo en importancia los Estados de México (cuatro casos), Guanajuato y Yucatán (tres casos en cada uno), y Aguascalientes, Chihuahua, Michoacán y San Luis Potosí (dos casos de cada Estado).

Lamentablemente, no se dispuso de información para ubicar el nivel de vulnerabilidad de la población inconforme con la atención médica, ya que, para el sistema de información y el esquema del expediente de laudos, no es obligatorio proporcionar información sobre nivel de escolaridad, ocupación ni adscripción a la seguridad social (en este caso de los padres de los menores afectados).

\section{Atención médica y padecimiento que originó la queja}

Resulta de vital importancia conocer el sector y la institución donde ocurrió el incidente que originó la presentación de la queja. Los datos disponibles dejan ver que en el $60 \%$ de los casos $(n=24)$ los menores fueron atendidos en el sector privado, en tanto que en el otro $40 \%$ ( $n=16)$ fueron atendidos en instituciones públicas de salud: siete en el Instituto de Seguridad y Servicios Sociales de los Trabajadores del Estado (ISSSTE), siete en la Secretaría de Salud (SSA) y dos en servicios estatales (Tabla 2).

Debe destacarse que el tipo de establecimiento donde se brindó la primera atención fue mayoritariamente una unidad hospitalaria: 27 de los 40 casos $(67.5 \%)$ acudieron inicialmente a un hospital en busca de atención, en tanto que 12 casos $(30 \%)$ iniciaron la atención del problema de salud en una unidad de consulta externa de primer nivel. Un establecimiento de apoyo (laboratorio) fue responsable del caso complementario.

\section{Principales servicios involucrados}

Como era de esperar, la mayor parte de las quejas de la población usuaria involucrada en las controversias que ahora se describen provinieron de los servicios de pediatría (17 casos, $42.5 \%$ ), seguidos por los servicios de urgencias y gineco-obstetricia, con cinco quejas (12.5\%); el tercer sitio correspondió a 
odontología, con cuatro quejas (10\%). Asimismo, aparecen tres casos provenientes tanto de cirugía general como de traumatología y ortopedia ( $7.5 \%$ cada uno). Llama la atención que la gran mayoría de los casos provenientes de pediatría fueron atendidos por establecimientos privados, así como la totalidad de los casos de odontología. En cuanto a los casos provenientes de los servicios de urgencias, la mayor parte fueron atendidos en unidades del sector público.

\section{Motivos de la queja}

Respecto a los motivos que originaron la controversia (denominada generalmente litis de la queja), estos pueden ser analizados bajo dos ópticas y diferentes niveles de desagregación, es decir, considerando solo la causa principal (de manera global y en grandes grupos) o bien tomando en cuenta el total de las causas con un mayor nivel de desagregación.

Considerando solo la razón principal (40 motivos enunciados) que aparece mencionada en el planteamiento general de la queja, el $45 \%$ de los demandantes (18 casos) hicieron mención de problemas relacionados con los auxiliares del diagnóstico; en particular, 13 denunciaron específicamente retrasos en la realización de determinados procedimientos. Con igual peso relativo (20\%) aparecieron motivos vinculados a la atención al parto y al puerperio (ocho casos), así como motivos relacionados con el diagnóstico (cinco de ocho causas). Otros tres casos registraron complicaciones posoperatorias dentro del grupo relativo a tratamientos, por mencionar los más importantes.

El total de motivos mencionados en la queja es de 209, es decir, un promedio de 5.2 causas por caso analizado (Tabla 3).

\section{Morbilidad origen del incidente que motivó la queja}

La morbilidad presentada por la población usuaria de los sistemas de salud, cuya atención médica dio lugar a la presentación de una queja, se presenta según los grandes capítulos de la Clasificación Internacional de Enfermedades. La mayor frecuencia de casos corresponde a enfermedades del aparato digestivo $(n=8)$, seguida de ciertas afecciones originadas en el periodo perinatal $(n=5)$, así como de las enfermedades del sistema respiratorio $(n=4)$ y las malformaciones congénitas $(n=4)$ (Tabla 4).

\section{Seguridad del paciente}

Cuando se analiza la calidad de la atención médica, uno de los componentes clave debe ser la seguridad del paciente. Los incidentes que ocurren en el ámbito hospitalario impactan en grados diversos tanto a la población como al médico involucrado, e incluso a la credibilidad misma del sistema de salud en su conjunto, no solo desde el punto de vista sanitario, sino también desde el económico, el social e, incluso, el jurídico ${ }^{7}$. Al hablar de los incidentes adversos ${ }^{c}$, se consideran como aquellos eventos en los que se produjo, o pudo haberse provocado, un daño no intencionado al paciente ${ }^{8}$.

Cabe mencionar una importante limitación en el análisis que ahora se presenta, ya que se carece de denominadores que encuadren este problema en el contexto nacional o en un ámbito más global del sistema de salud; por ello, este apartado se limita a describir las categorías de los incidentes adversos encontrados en los expedientes de laudos concluidos y su distribución porcentual, y a mostrar un indicador sobre la incidencia relativa de cada rubrod (Tabla 5 ).

Para el caso del presente análisis, en relación con las 40 quejas resueltas a través del arbitraje médico dentro del periodo 2011-2015, se encontraron en total 351 incidentes o eventos adversos, lo que hace un promedio de aproximadamente 8.8 por caso. Dichos incidentes fueron organizados según una lista abreviada de eventos

c Según la Clasificación Internacional de Seguridad del Paciente, un incidente adverso es un evento que cumple uno o más criterios de los que a continuación se enlistan: 1) un paciente ha resultado lesionado, con independencia de la posible responsabilidad del hospital; 2) el ingreso ha sido consecuencia de un resultado adverso de la atención ambulatoria; 3) el paciente ha sido ingresado de nuevo por complicaciones o por una atención incompleta en el ingreso anterior; 4) ha habido deficiencias en la documentación, por ejemplo, en los procedimientos de consentimiento informado o en la historia clínica; 5) se ha llevado a cabo una intervención quirúrgica no prevista; 6) se han empleado procedimientos que no cumplían los criterios de idoneidad del hospital; 7) ha surgido un problema con el uso de sangre o hemoderivados; 8) se ha producido una infección nosocomial (contraída en el hospital); 9) se han utilizado fármacos incorrectamente; 10) se ha producido un paro cardiaco o respiratorio o la muerte; 11) ha ocurrido un incidente (como una caída del paciente); 12) no se ha hecho un seguimiento de datos radiológicos o de laboratorio anómalos; 13) la estancia ha sido anormalmente breve o larga para el trastorno; 14) ha habido problemas para obtener servicios; y 15) el paciente o la familia se han mostrado insatisfechos.

d A manera de contexto general, vale la pena mencionar que un estudio realizado en dos hospitales del sector público durante 2006 señaló una prevalencia del $11.8 \%$ de eventos adversos respecto al total de egresos ${ }^{9}$. 
Tabla 3. Motivos mencionados en el momento de presentación de la queja, según grandes grupos desagregados, período 2011-2015

\begin{tabular}{|c|c|c|c|}
\hline Código & Motivos de la queja según laudos concluidos & Total & Porcentaje \\
\hline & Atención de parto y puerperio & 28 & 13.4 \\
\hline 1.1 & Accidentes e incidentes & 2 & 1 \\
\hline 1.3 & Atención del parto por personal no calificado & 2 & 1 \\
\hline 1.4 & Complicaciones del embarazo a la madre & 3 & 1.4 \\
\hline 1.5 & Complicaciones del embarazo al producto & 1 & 0.5 \\
\hline 1.6 & Complicaciones del parto a la madre & 1 & 0.5 \\
\hline 1.7 & Complicaciones del parto al producto & 1 & 0.5 \\
\hline 1.8 & Complicaciones del puerperio & 2 & 1 \\
\hline 1.9 & Falta de control prenatal o control inadecuado & 2 & 1 \\
\hline 1.13 & Falta de seguimiento o seguimiento inadecuado del puerperio & 3 & 1.4 \\
\hline 1.15 & Retraso o negativa de la atención del parto & 2 & 1 \\
\hline 1.16 & Seguimiento inadecuado del trabajo de parto & 5 & 2.4 \\
\hline 1.17 & Técnica inadecuada & 2 & 1 \\
\hline \multirow[t]{2}{*}{1.18} & Valoración inadecuada al egreso hospitalario & 2 & 1 \\
\hline & Auxiliares del diagnóstico & 27 & 12.9 \\
\hline 2.2 & Estudios innecesarios & 3 & 1.4 \\
\hline 2.3 & Falsos positivos o negativos & 4 & 1.9 \\
\hline 2.5 & Retraso del procedimiento diagnóstico & 17 & 8.1 \\
\hline 2.6 & Retraso o falta de notificación de resultados & 1 & 0.5 \\
\hline \multirow[t]{2}{*}{2.7} & Secuelas & 2 & 1 \\
\hline & Diagnóstico & 47 & 22.5 \\
\hline 3.1 & Desinformación sobre el diagnóstico & 7 & 3.3 \\
\hline 3.2 & Diagnóstico erróneo & 16 & 7.7 \\
\hline 3.3 & Omisión de diagnóstico & 6 & 2.9 \\
\hline \multirow[t]{2}{*}{3.4} & Retardo en el diagnóstico & 18 & 8.6 \\
\hline & Tratamiento médico & 47 & 22.5 \\
\hline 4.1 & Accidentes e incidentes & 2 & 1 \\
\hline 4.2 & Complicaciones secundarias & 8 & 3.8 \\
\hline 4.3 & Desinformación sobre el tratamiento & 1 & 0.5 \\
\hline 4.4 & Falta de consentimiento & 1 & 0.5 \\
\hline 4.5 & Retraso del tratamiento & 12 & 5.7 \\
\hline 4.6 & Secuelas: exceso terapéutico & 3 & 1.4 \\
\hline 4.7 & Tratamiento inadecuado o innecesario & 13 & 6.2 \\
\hline 4.8 & Tratamiento insatisfactorio & 6 & 2.9 \\
\hline 4.9 & Otros & 1 & 0.5 \\
\hline
\end{tabular}


Tabla 3. Motivos mencionados en el momento de presentación de la queja, según grandes grupos desagregados, período 2011-2015 (Continuación)

\begin{tabular}{|c|c|c|c|}
\hline Código & Motivos de la queja según laudos concluidos & Total & Porcentaje \\
\hline & Tratamiento quirúrgico & 30 & 14.4 \\
\hline 5.1 & Accidentes e incidentes & 4 & 1.9 \\
\hline 5.2 & Alta prematura de los cuidados posoperatorios & 2 & 1 \\
\hline 5.3 & Cirugía innecesaria & 4 & 1.9 \\
\hline 5.4 & Complicaciones quirúrgicas del posoperatorio & 1 & 0.5 \\
\hline 5.6 & Error quirúrgico & 1 & 0.5 \\
\hline 5.8 & Falta de seguimiento o seguimiento inadecuado en el posoperatorio & 2 & 1 \\
\hline 5.9 & Falta de valoración prequirúrgica & 6 & 2.9 \\
\hline 5.10 & Retraso del tratamiento quirúrgico & 5 & 2.4 \\
\hline 5.11 & Secuelas & 2 & 1 \\
\hline \multirow[t]{2}{*}{5.12} & Técnica quirúrgica inadecuada & 3 & 1.4 \\
\hline & Relación médico-paciente & 20 & 9.6 \\
\hline 6.1 & Desinformación sobre el diagnóstico & 7 & 3.3 \\
\hline 6.2 & Fallas en la comunicación & 12 & 5.7 \\
\hline \multirow[t]{2}{*}{6.3} & Trato inadecuado & 1 & 0.5 \\
\hline & Deficiencias administrativas & 10 & 4.8 \\
\hline 7.1 & Cambio de médico tratante o de unidad médica & 1 & 0.5 \\
\hline 7.2 & Demora prolongada o diferimiento para obtener el servicio & 1 & 0.5 \\
\hline 7.3 & Falta de equipo médico & 3 & 1.4 \\
\hline 7.4 & Falta de insumo o medicamentos & 1 & 0.5 \\
\hline 7.7 & Sistema de referencia y contrarreferencia & 2 & 1 \\
\hline 7.8 & Trato inadecuado por personal administrativo & 1 & 0.5 \\
\hline \multirow[t]{2}{*}{7.9} & Otros & 1 & 0.5 \\
\hline & Total & 209 & 100 \\
\hline
\end{tabular}

adversos (siete grandes grupos), misma que se desagrega en un poco más de 84 categorías (Tabla 3).

De acuerdo con la organización mencionada, destacan los siguientes resultados:

- La mayor frecuencia de eventos adversos correspondió a los problemas relacionados con procedimientos (91 incidentes), lo que representa el $25.9 \%$ del total y una incidencia relativa de 127 incidentes por cada 100 casos. En particular, destacaron en este grupo las categorías de tratamiento médico ineficiente 0 ineficaz, el no haber agotado los medios de tratamiento y la intervención quirúrgica incompleta o ineficaz.
- Siguieron los incidentes relacionados con problemas de medicación ( $n=65$ ), concentrando el $18.5 \%$ del total de incidentes, lo que implica 162 incidentes por cada 100 quejas presentadas. Las categorías más frecuentemente reportadas fueron la preparación inadecuada del medicamento, los errores en la manipulación de este, el retraso en su administración y la incorrecta monitorización del medicamento. Llama la atención que en la literatura internacional siempre aparece este grupo de incidentes como el de mayor frecuencia en los pacientes pediátricos, lo que no sucede con los presentes datos; la razón pudiera ser que el usuario (o sus familiares en este caso) no 
Tabla 4. Causas de morbilidad en los 40 laudos analizados

\begin{tabular}{|l|l|c|c|}
\hline Grupo & Causas de morbilidad por grupo & Casos (n) & Porcentaje \\
\hline I & Ciertas enfermedades infecciosas y parasitarias & 2 & 5 \\
\hline II & Neoplasias & 2 & 5 \\
\hline IV & Enfermedades endocrinas, nutricionales y metabólicas & 1 & 2.5 \\
\hline IX & Enfermedades del sistema circulatorio & 1 & 2.5 \\
\hline VI & Enfermedades del sistema nervioso & 2 & 5 \\
\hline VII & Enfermedades del ojo y sus anexos & 1 & 2.5 \\
\hline X & Enfermedades del sistema respiratorio & 4 & 10 \\
\hline XI & Enfermedades del aparato digestivo & 8 & 20 \\
\hline XIII & Enfermedades del sistema osteomuscular y del tejido conectivo & 2 & 5 \\
\hline XIV & Enfermedades del aparato genitourinario & 2 & 5 \\
\hline XIX & Traumatismos, envenenamientos y algunas otras consecuencias de causa externa & 3 \\
\hline XVI & Ciertas afecciones originadas en el periodo perinatal & 5 \\
\hline XVII & Malformaciones congénitas, deformidades y anomalías cromosómicas & 7.5 \\
\hline XVIII & Síntomas, signos y hallazgos anormales, no clasificados en otra parte & 12.5 \\
\hline XXI & Factores que influyen en el estado de salud y contacto con los servicios de salud & 1 & 10 \\
\hline & Total & 40 \\
\hline
\end{tabular}

Tabla 5. Incidentes adversos según grandes categorías en los 40 laudos analizados, periodo 2011-2015

\begin{tabular}{|l|l|c|c|c|}
\hline Código & Eventos adversos & Total & Distribución porcentual & Incidencia* \\
\hline $\mathrm{Ge}$ & Incidentes adversos relacionados con la gestión & 51 & 14.5 & 127.5 \\
\hline $\mathrm{Co}$ & Incidentes adversos relacionados con la comunicación & 46 & 13.1 & 115 \\
\hline $\mathrm{Di}$ & Incidentes adversos relacionados con el diagnóstico & 50 & 14.2 & 125 \\
\hline $\mathrm{Pr}$ & Incidentes adversos relacionados con los procedimientos & 91 & 25.9 & 227.5 \\
\hline $\mathrm{Me}$ & Incidentes adversos relacionados con los medicamentos & 65 & 18.5 & 162.5 \\
\hline $\mathrm{Cu}$ & Incidentes adversos relacionados con los cuidados & 37 & 10.5 & 92.5 \\
\hline $\mathrm{In}$ & Incidentes adversos relacionados con las infecciones & 11 & 3.1 & 27.5 \\
\hline & Total & 351 & 100 & 877.5 \\
\hline
\end{tabular}

*El denominador de la incidencia corresponde a los 40 casos analizados.

siempre se da cuenta de lo ocurrido, y en el relato de hechos no necesariamente se menciona. De igual forma, en la documentación incluida por los prestadores, el énfasis se pone en los procedimientos realizados y no tanto en los detalles del proceso, como podría ser la medicación, generalmente a cargo del personal de enfermería, que no en todos los casos presenta sus hojas de control.
- En seguida aparecieron los incidentes originados por la gestión administrativa $(n=51)$, representando el $14.5 \%$ de los eventos globales, lo que implicaría, en términos de incidencia relativa, que ocurrieran 127 por cada 100 casos similares. Las principales categorías mencionadas en este grupo se refieren a problemas ante un expediente incompleto, incluyendo la pérdida de documentación, seguida de una lista de espera prolongada. 
- Con una frecuencia cercana a la del grupo anterior, se encontraron los incidentes adversos relacionados con el diagnóstico $(n=50)$, que significan el $14.2 \%$ de la cifra total de eventos y que implican, en términos relativos, una ocurrencia potencial de 125 incidentes por cada 100 inconformidades o quejas. Las categorías que destacaron en forma relevante fueron no haber agotado los medios para el diagnóstico, el error en el diagnóstico y el retraso en la detección de este.

- En el quinto sitio $(n=46)$ aparecieron los problemas de comunicación entre el personal médico y los familiares o con el personal de enfermería, e incluso entre dos o más médicos. Este grupo concentró el $13.1 \%$ de los eventos totales y su incidencia relativa fue de 115 eventos adversos por cada 100 quejas presentadas.

- En el penúltimo sitio $(n=37)$ se encontraron los incidentes adversos relacionados con los cuidados al menor, representando un poco más del $10 \%$ del total de los incidentes registrados en forma global; su incidencia relativa implicó esperar la ocurrencia de 93 eventos por cada 100 quejas. En el caso de la población pediátrica destacó solo una categoría, la referente al seguimiento inadecuado de la evolución del paciente.

- El grupo de infecciones asociadas con la salud apareció con el menor número de casos $(n=11)$, aun cuando, junto con la frecuencia, implicó la mayor letalidad, en particularmente en las edades más jóvenes de este estrato poblacional. Representó el $3.1 \%$ de los casos, con una incidencia relativa de 28 eventos infecciosos por cada 100 quejas analizadas. Destacaron las categorías de infección de la herida quirúrgica, sepsis y choque séptico, así como neumonía nosocomial.

\section{Daños sufridos a consecuencia de la presunta atención médica}

\section{Defunciones}

De acuerdo con los documentos integrados en los expedientes médico-legales conformados en ocasión de las 40 quejas objeto del presente estudio, presuntamente a consecuencia de la concatenación de uno 0 varios de los eventos adversos antes mencionados, ocurrieron 12 defunciones de menores de 15 años (siete de sexo masculino y cinco de sexo femenino, la mitad de ellos menores de 1 año) durante el periodo de análisis. Por su gravedad, estas muertes son
Tabla 6. Tipo de discapacidad sufrida por los usuarios de los servicios de salud en los 40 laudos analizados, periodo 2011-2015

\begin{tabular}{|l|c|c|}
\hline Tipo de discapacidad & Total $(\mathbf{n})$ & Porcentaje \\
\hline Discapacidad motriz & 7 & 50 \\
\hline $\begin{array}{l}\text { Discapacidad sensorial y de la } \\
\text { comunicación }\end{array}$ & 4 & 28.6 \\
\hline Discapacidades múltiples & 1 & 7.1 \\
\hline Discapacidades no especificadas & 2 & 14.3 \\
\hline Total & 14 & 100 \\
\hline
\end{tabular}

consideradas como presuntos eventos centinela ${ }^{e}$. La relación entre el número de fallecimientos respecto al total de casos arroja un índice muy alto, que implica una incidencia relativa de 30 defunciones por cada 100 casos analizados (12/40), lo que relativiza la gravedad del problema.

Entre las causas de muerte que ocasionaron las defunciones señaladas se encuentran algunas vinculadas a enfermedades del sistema circulatorio (cuatro casos), del sistema respiratorio (un caso) y del sistema nervioso (un caso); enfermedades endocrinas, nutricionales y metabólicas (un caso); ciertas afecciones en el periodo perinatal, con neoplasias malignas (un caso) y algunas otras de carácter poco especificado (tres casos).

\section{PRESENCIA Y TIPO DE DISCAPACIDAD}

Respecto a las consecuencias sufridas por la presunta mala atención recibida, además de las 12 defunciones antes señaladas, en 14 expedientes se reportaron casos de discapacidad, tal como se muestra en la tabla 6. En los otros 14 casos, los afectados no hicieron mención de este tipo de problemas.

Como puede observarse, en la mitad de los casos se reportaron discapacidades motrices $(n=7)$, seguidas de las discapacidades de tipo sensorial y de la comunicación $(n=4)$. Un caso mencionó una discapacidad múltiple y en dos casos, si bien se mencionó el problema, no se especificó a qué discapacidad se refería (Tabla 6).

A partir de estas cifras puede estimarse una incidencia respecto a la gravedad del daño sufrido por algún

e Se considera como un evento centinela un hecho inesperado resultante de la atención médica, que produce la muerte o la pérdida de una función o de un órgano. 
Tabla 7. Resolución del laudo en los 40 casos analizados, periodo 2011-2015

\begin{tabular}{|l|c|c|c|c|c|}
\hline \multirow{2}{*}{ Institución involucrada } & \multicolumn{2}{|c|}{ Evaluación del acto médico } & \multirow{2}{*}{ Total laudos } & \multicolumn{2}{c|}{ Conclusión del laudo } \\
\cline { 2 - 6 } & $\begin{array}{c}\text { Con evidencia de mala } \\
\text { práctica }\end{array}$ & $\begin{array}{c}\text { Sin evidencia de mala } \\
\text { práctica }\end{array}$ & & Condenatorio & Absolutorio \\
\hline SSA & 6 & 1 & 7 & 6 & 1 \\
\hline Servicios estatales & 5 & 2 & 7 & 2 & 0 \\
\hline ISSSTE & 2 & 0 & 2 & 11 & 13 \\
\hline Privados & 13 & 11 & 24 & 24 & 16 \\
\hline Total & 26 & 14 & 40 & 24 \\
\hline
\end{tabular}

ISSSTE: Instituto de Seguridad y Servicios Sociales de los Trabajadores del Estado; SSA: Secretaría de Salud.

tipo de discapacidad, cifra que asciende a 35 casos por cada 100 quejas analizadas (14/40). Dicha cifra se modifica ligeramente si se toma en cuenta que son nueve los casos calificados con discapacidad permanente, quedando la incidencia de discapacidad permanente en 22.5 casos por cada 100 quejas recibidas.

Un indicador muy general sobre la presencia del daño estaría dado por la consideración de la presencia de muerte o de discapacidad permanente en los casos de laudos concluidos (21/40), lo que indica que 52 de cada 100 usuarios menores de 15 años que se sometieron al proceso arbitral en CONAMED sufrieron discapacidad permanente o muerte.

\section{Evaluación del acto médico y conclusión del laudo}

Una de las fases sustantivas del proceso de revisión y análisis de la documentación probatoria entregada a CONAMED por las partes involucradas en el conflicto médico-legal lo constituye el proceso de evaluación del acto médico, a fin de poder encontrar elementos objetivos sobre la evidencia o no de mala práctica. La importancia de esta fase reside en que es el momento del proceso en el que se determina si los daños o hechos clínicos relatados o documentados por las partes involucradas son aspectos inherentes a la evolución natural de la enfermedad, si se deben a omisiones 0 incumplimientos del tratamiento por parte del paciente o si son consecuencias del acto médico en sí.

En este sentido, de la revisión de los 40 laudos de menores de 15 años provenientes de los servicios de pediatría concluidos durante el periodo 2011-2015, se determinó que 26 de ellos (65\%) sí contaban con elementos de mala práctica médica, en tanto que 14 no mostraron tales evidencias (35\%) (Tabla 7).
El comportamiento institucional resulta interesante, pues si bien la mitad de los laudos con evidencia de mala práctica corresponden al sector privado, la proporción respecto al total dentro de ese mismo sector resultó la más baja de todas, con el 54\% (es decir, 13 de 24), en tanto que tal indicador aumentó al $71.4 \%$ para el ISSSTE y al $88.9 \%$ para la SSA y los servicios estatales considerados en forma conjunta.

Si bien la evaluación documental sobre el acto médico es de suma importancia para el dictamen de la sentencia final por parte de los peritos institucionales, la conclusión del laudo no se da en forma automática a partir de la determinación previa, pues se consideran dichos resultados a la luz de la demanda concreta de los demandantes, pudiendo ocurrir que la mala práctica se encuentre desvinculada de la litis (o motivo principal) de la queja; a eso se le llama mala práctica no vinculatoria.

Según la sentencia final emitida en relación con los 40 laudos, el $60 \%(n=24)$ recibió el veredicto de condenatorio, en tanto que el $40 \%$ restante $(n=16)$ fueron laudos absolutorios. Consecuencia inmediata de ello es que mientras en el primer caso los demandantes tienen derecho de recibir el pago de las indemnizaciones o reembolsos solicitados, en el caso de los resolutivos absolutorios se exime al prestador de servicios de tener que cubrir las pretensiones demandadas por el usuario en cuestión.

Cabe señalar que en algunos casos el proceso no concluye con el dictamen de la sentencia, pues ocurre que el demandante (o el demandado) están en su derecho de inconformarse con la determinación institucional tomada, y en ese caso proceden a la presentación de un amparo. Tal es el caso de siete de los 40 laudos concluidos, de los cuales se ampararon tres en relación con la SSA, tres contra el ISSSTE y uno contra los servicios estatales de salud. 


\section{Discusión}

A manera de conclusión puede decirse que, si bien no existen elementos objetivos para afirmar que la seguridad del paciente pediátrico ha mejorado en los últimos años, por no existir información que documente tal comportamiento, podría inferirse que cada vez se toma más en cuenta el conocimiento de los incidentes adversos que afectan de manera real o potencial a la población usuaria de los servicios de salud en México ${ }^{9-12}$. Así, surge la necesidad de promover la difusión y el conocimiento de este tema y los conceptos que le son propios, tanto en el área de la pediatría como en cualquier otro servicio o especialidad. El personal médico y de salud debe conocer los riesgos y las consecuencias en que derivan estos incidentes, para que se preste especial atención, en todos los contextos, a la relación médico-paciente. Con ello, no solo mejorará la calidad del servicio prestado, sino que se evitarán inconformidades y quejas, así como los daños físicos y emocionales que ello conlleva, no solo para la población usuaria y su entorno más cercano, sino también para los médicos y el personal de salud como segundas víctimas de estos conflictos.

\section{Responsabilidades éticas}

Protección de personas y animales. Los autores declaran que para este estudio no se han realizado experimentos en seres humanos ni en animales.

Confidencialidad de los datos. Los autores declaran que en este artículo no aparecen datos de pacientes.

Derecho a la privacidad y consentimiento informado. Los autores declaran que en este artículo no aparecen datos de pacientes.

\section{Agradecimientos}

Al Dr. Rafael Rodríguez, por su apoyo en el manejo de la información.

\section{Financiamiento}

\section{Ninguno.}

\section{Conflicto de intereses}

Los autores declaran no tener ningún conflicto de intereses.

\section{Bibliografía}

1. Comisión Nacional de Arbitraje Médico. México: Proyecto Ariel. Marco conceptual y documentos técnicos que lo integran. 2017. Disponible en: http://www.conamed.gob.mx/publicaciones/ariel.php

2. Hernández F, Aguilar MT, Santacruz Varela J, Rodríguez Martínez Al, Fajardo Dolci GE. Queja médica y la calidad de la atención. Rev CONAMED. 2009;14:26-34.

3. Aguirre Gas H, Campos Castolo EM, Carrillo Jaimes A, Zavala Suárez E, Fajardo Dolci GE. Análisis crítico de la queja médica. Rev CONAMED. 2008:13:5-16.

4. World Health Organization. The conceptual framework for the International Classification for Patient Safety Version 1.0 for use in field testing 2007-2008. WHO; 2017. Disponible en: http://www.who.int/patientsafety/ ICPS\%20-\%20July\%202007.pdf.

5. Ley del Seguro Social, Artículo 296 (reformado 20-12-2001). Diario Oficial de la Federación.

6. Secretaría de Salud. Modelo de Arbitraje Médico. México; 2017. Disponible en: http://www.salud.gob.mx/unidades/cdi/documentos/DOCSAL7339.pdf

7. Estudio Nacional sobre los efectos adversos ligados a la hospitalización (ENEAS), 2005. Dirección General de la Agencia de Calidad del Sistema Nacional de Salud. Reporte 2006. Madrid: Ministerio de Sanidad y Consumo; 2006.

8. Lezana MA, Fernández S, Rodríguez J. Laudos 2007-2011: aprendiendo sobre los incidentes adversos y otras características de la queja médica. Rev CONAMED. 2015;20:101-10.

9. Observatorio del Desempeño Hospitalario 2006. Secretaría de Salud/ Dirección General de Evaluación del Desempeño. México; 2007. Sede web: www.dged.salud.gob.mx

10. Infante C. Quejas médicas: la insatisfacción de los pacientes con respecto a la calidad de la atención médica. México: Editores de Textos Mexicanos; 2006

11. Dubón MC, Romero ME. Análisis de controversias en arbitraje médico. Rev CONAMED. 2012;17:100-8.

12. Bustamante-Leija LE, Maldonado-Camargo VM, González-Anaya C, Gutiérrez Vega R. Mecanismos alternativos de solución de controversias en la prestación de servicios de salud. Rev CONAMED. 2012;17:126-9. 